\title{
Notice of Duplicate Publication
}

Hiroshi Yamamoto, Shinji Teramoto, Yasuhiro Yamaguchi, Yasuyoshi Ouchi: Effect of nasal continuous positive airway pressure treatment on plasma adrenomedullin levels in patients with obstructive sleep apnea syndrome: roles of nocturnal hypoxia and oxidant stress. Hypertens Res 2007; 30: 1065-1076.

The above article published in Volume 30, No. 11 (November) is a duplicate publication published in Sleep Medicine as follows:

Hiroshi Yamamoto, Shinji Teramoto, Yasuhiro Yamaguchi, Yoko Hanaoka, Masaki Ishii, Shinichiro Hibi, Yasuyoshi Ouchi: Long-term oxygen administration reduces plasma adrenomedullin levels in patients with obstructive sleep apnea syndrome. Sleep Med 2007; 9: 80-87.

With this notification, Hypertension Research is now withdrawing the article published in its 2007; 30 (11) issue.

\section{Note from the Editor-in-Chief}

Duplicate publication of essentially the same data constitutes a violation of copyright law and of the ethics of scientific publication. When there is doubt about the common source, overlapping, or coincidence of data in articles that are based on the same study, the author(s) must disclose any information about their contribution being under consideration or accepted by another journal at the time of submission of a manuscript.

\author{
Kazuyuki Shimada \\ Editor-in-Chief \\ Hypertension Research
}

